\title{
Treatment of esophageal-gastric double primary cancer by pedunculated remnant gastric interposition, esophageal-gastric anastomosis and gastrojejunal Billroth II anastomosis: A case report
}

\author{
XIAO TIAN ZHANG ${ }^{1}$, WEI WANG ${ }^{2}$, QIANG ZHU ${ }^{2}$, MING CAO $^{2}$, ZHONG MIN JIANG $^{2}$ and QI ZANG $^{2}$ \\ ${ }^{1}$ Graduate Department of Weifang Medical College, Weifang, Shandong 261053; ${ }^{2}$ Department of Thoracic Surgery, \\ Qianfoshan Hospital Affiliated to Shandong University, Jinan, Shandong 250014, P.R. China
}

Received July 22, 2014; Accepted April 20, 2015

DOI: $10.3892 / \mathrm{ol} .2015 .3301$

\begin{abstract}
With the continuous advancement of clinical diagnostic techniques, including imaging technology, the incidence of confirmed multiple primary cancers or double primary carcinoma increases yearly. However, studies reporting synchronization surgery performed for primary dual esophageal gastric cancer are rare. The present study reports the case of a patient with double primary esophageal-gastric cancer, located in the thoracic cavity segment of the esophagus and gastric antrum of the stomach, respectively. The gastric cancer was diagnosed by endoscopy biopsy with concomitant esophageal cancer. The patient underwent gastric cancer resection, and pedunculated remnant gastric interposition esophagogastric side anastomosis was performed with gastrojejunostomy Billroth II anastomosis behind the colon. Abdominal cavity lymph node dissection was also performed. The esophagealgastric double primary cancer was simultaneously excised and the gastric regions were used in the construction of the upper gastrointestinal tract: The surgery was successful. However, two weeks after surgery, upper gastrointestinal imaging revealed esophagogastric anastomotic leakage. Subsequently, an esophageal stent was inserted and antibiotics and additional treatment was administered. Follow-up one year after surgery revealed that the patient was well and remained in a stable condition.
\end{abstract}

Correspondence to: Professor Qi Zang, Department of Thoracic Surgery, Qianfoshan Hospital Affiliated to Shandong University, 16766 Jingshi Road, Jinan, Shandong 250014, P.R. China

E-mail: qzang2005@sina.com

Key words: esophageal cancer, gastric cancer, esophagoplasty, esophagectomy

\section{Introduction}

Esophageal cancer and gastric cancer are common digestive tract tumors; however, the two cancers have a low incidence of becoming malignant. It has been reported that the incidence of multiple types of cancer associated with esophageal cancer is $8.3-12.6 \%$ (1), however the incidence of esophageal-gastric double primary cancer is lower at $0.8-1.5 \%$ (2). Due to the complexity of upper digestive tract reconstruction for double primary esophageal and stomach cancer, the literature rarely reports a case involving synchronized surgical resection.

Esophageal-gastric double primary cancer is difficult to diagnosis and the misdiagnosis rate is high, as it is often misdiagnosed as distant metastasis in the clinic (3). The treatment is often dominated by radiotherapy and chemotherapy, with less use of surgery. Surgery may be used to first remove the tumor if it is relatively advanced and then an appropriate tissue for reconstruction of the upper digestive tract, including the as stomach or intestine, is selected. The double primary cancer resection surgery is complicated, resulting in large injury for the patient; the reconstruction of the digestive tract is difficult, and may lead to tissue necrosis and other complications. The esophageal or gastric cancer resection surgery alone is relatively simple, often with remnant gastric of upper digestive tract reconstruction, the arising complications of this surgery are usually less than those observed in a double primary cancer resection (4).

The present study reports the case of a patient that underwent synchronized surgical resection for the treatment of esophageal-gastric double primary cancer. The post-operative recovery of the patient was monitored.

\section{Case report}

The study was approved by the ethics committee of Qianfoshan Hospital Affiliated to Shandong University (Jinan, P.R. China). A 58-year-old male patient presented with paroxysmal abdominal pain of a 40-day duration. Physical examination revealed no positive signs: There was no anemia or superficial node enlargement or enlargement of the liver or spleen. In addition, the laboratory examination results 

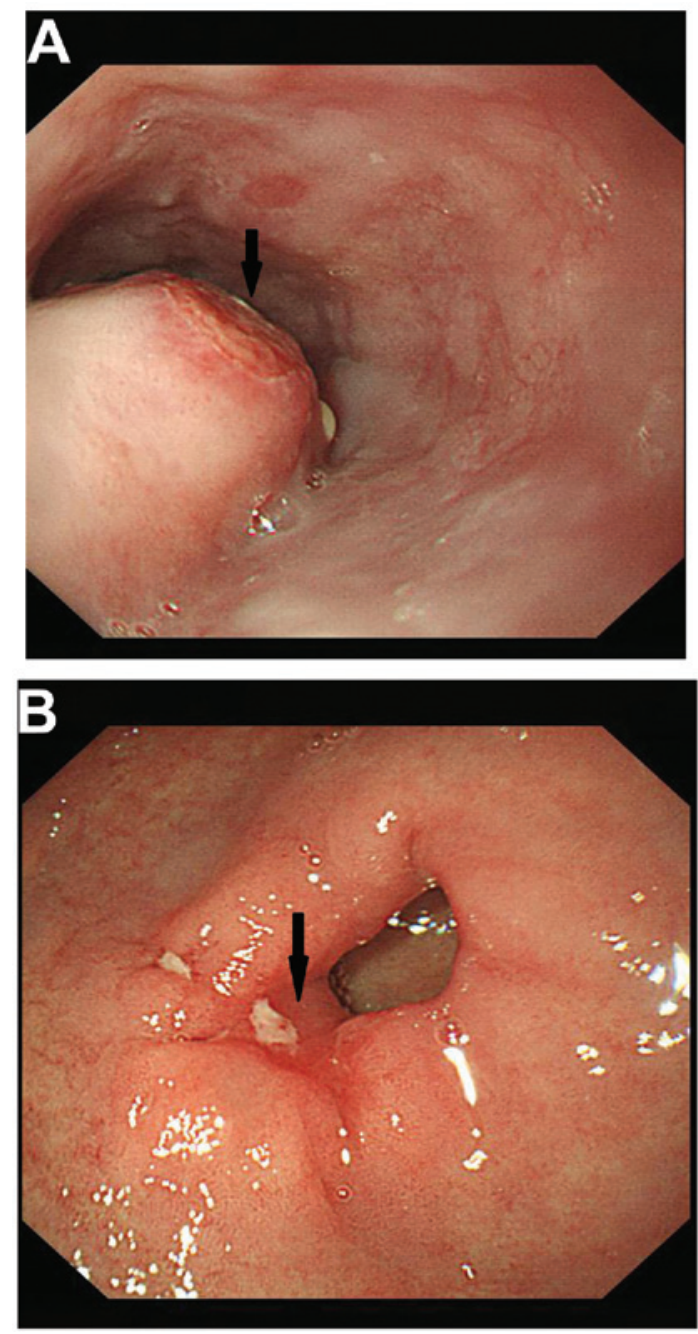

Figure 1. Endoscopy revealed the lesions of the (A) esophageal and (B) gastric cancers.

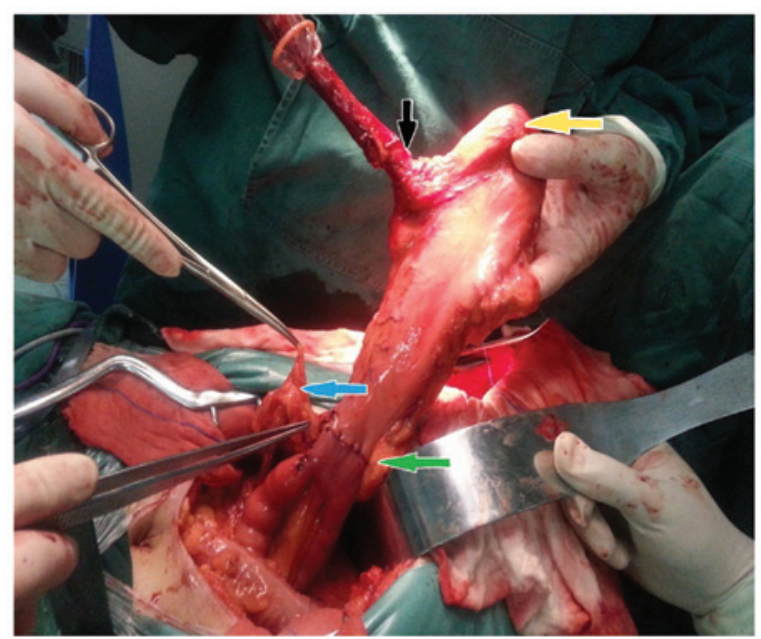

Figure 2. Intraoperative image revealing the fundus of the stomach (yellow arrow), esophagus (black arrow), right gastroepiploic artery (blue arrow) and esophagogastric anastomosis (green arrow).

were within the normal range: White blood cell count, $6.57 \times 10^{9} / 1\left(3.97-9.15 \times 10^{9} / 1\right)$; Red blood cell count, $5.04 \times 10^{12} / 1$ (4.09-5.74x1012/1); hemoglobin, $150 \mathrm{~g} / 1$ (131-172 g/l); alanine
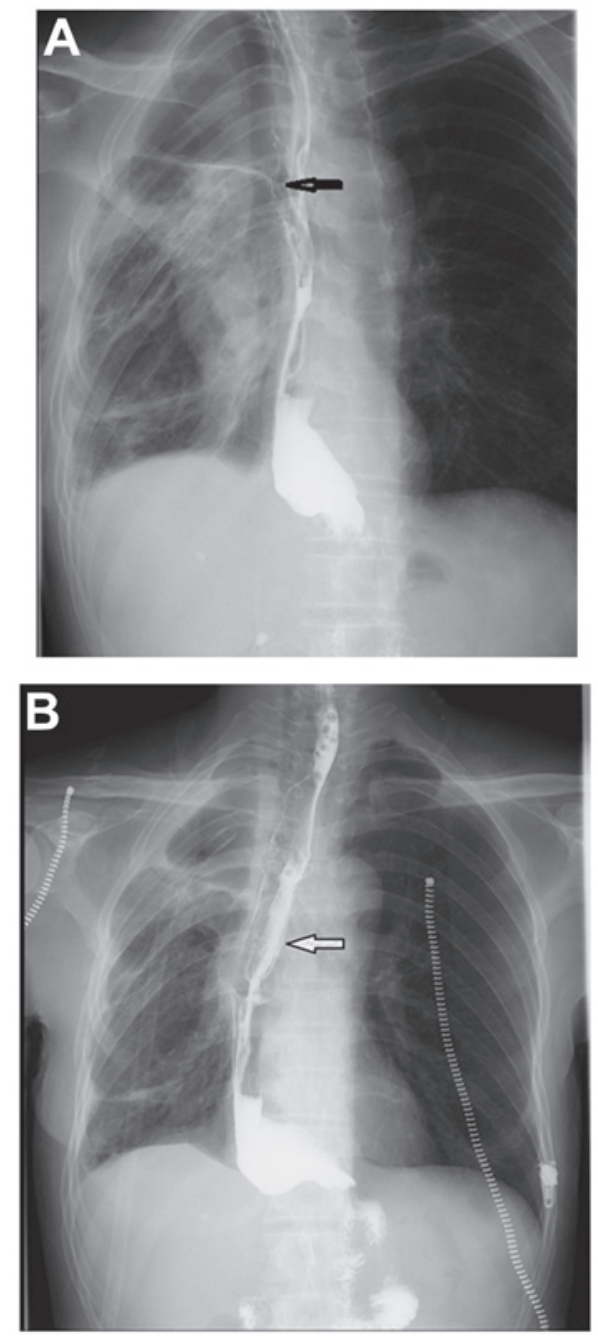

Figure 3. Upper gastrointestinal imaging revealing (A) the esophagogastric anastomotic leakage and (B) the esophageal stent that was inserted as a result.

aminotransferase, $19.9 \mathrm{U} / 1$ (8-40 U/1); aspartate aminotransferase, $12.5 \mathrm{U}$ (5-49 U/l); albumin, $39.3 \mathrm{~g} / 1$ (35-55 g/l); and blood urea nitrogen, $86.8 \mu \mathrm{mol} / 1(59-109 \mu \mathrm{mol} / \mathrm{l})$. Chest and upper abdominal computed tomography (CT) revealed thickening in the middle and lower segments of the esophageal wall, which was consistent with CT findings of esophageal cancer. Right lung inflammation, with slight dilation of the right middle lobe bronchus, emphysema of the lungs, and enlarged subcarinal lymph nodes were also identified by CT, whereas the abdominal cavity lymph nodes were not observed as enlarged. Endoscopy was performed using an Olympus CLV-290SL endoscope (Olympus Corporation, Tokyo, Japan), and revealed space-occupying lesions in the esophagus and gastric antrum (Fig. 1). The biopsy revealed esophageal squamous cell carcinoma. Severe dysplasia and carcinoma in situ was observed in the pyloric portion of the gland, which did not exclude early invasion. The diagnosis of esophageal-gastric double primary cancer was made.

In March 2013, it was observed that the esophageal tumor had invaded the tunica adventitia through the right thoracic wall, and therefore, the patient underwent tumor separation and cleaning of the swollen lymph nodes. An upper abdominal incision was performed for abdominal exploration. It was 
found that the tumor, which was adjacent to the pyloric ring, did not invade the serosa. Therefore, distal portion gastrectomy and Billroth stomach anastomosis behind the colon were performed. Subsequently, esophageal cancer resection and gastroesophageal anastomosis in the right chest were also performed.

The gastrocolic ligaments, blood vessels and lymph nodes around the stomach were dissociated to retain the right gastroepiploic artery (Fig. 2). The gastric tissue was then dissociated, the gastric antrum was dissected to expose the proximal duodenum, the duodenum was excised where diseased and the remnant duodenum was closed. Finally, the proximal gastric tumor of the gastric cancer was resected. Remnants of the gastric cancer cells were not observed in the intraoperative rapid frozen sections. Subsequently, gastrojejunostomy side-side anastomosis (Fig. 2) behind the colon was performed with Billroth surgery completion. The esophagus was cut, with margins of $>5 \mathrm{~cm}$ from the edge of the esophageal cancer lesion. The lesser curvature of the stomach tissue was removed to form a tubular stomach, and then proximal anastomosis of the esophagus and gastric posterior wall was performed. The stomach drainage and duodenum feeding tubes were placed, and the stomach was sent into the esophageal mediastinal bed prior to the upper mediastinal pleura being sutured. The thorax was flushed and no active bleeding was identified. The indwelling pleural and peritoneal drainage tube was removed, and the thoracic and abdominal incision was sutured to complete the surgery.

Post-operative pathology revealed the lesion to be moderately differentiated esophageal squamous cell carcinoma, the majority of which was autolytic. The lesion was a medullary type tumor with a volume of $4.5 \times 0.6 \mathrm{~cm}$ that had achieved deep myometrial invasion. Metastasis of the cancer was not found during the lymph node check-up. The antral glands were involved in the high-grade intraepithelial neoplasia, consisting of severe dysplasia and carcinoma in situ, due to local early infiltration and invasion of the muscularis mucosa. Cancer was not found in the regional lymph nodes. The esophageal cancer was classified as a moderately differentiated middle thoracic section squamous cell carcinoma, with a tumor-node metastasis (TNM) stage of pT2N0M0. The gastric cancer lesion was located in the antrum of the stomach and was classified as TNM stage T1N0M0b [Union for International Cancer Control TNM staging, 2009 edition (5)].

Two weeks subsequent to the surgery, upper gastrointestinal imaging of the patient revealed esophagogastric anastomotic leakage, as evidenced by the presence of barium used for imaging in the chest (Fig. 3A). Two possible causes were considered, poor blood flow to the lower stomach or the onset of post-operative pulmonary infection, leading to bad atelectasis. Therefore, an esophageal stent was inserted immediately (Fig. 3B), and effective antibiotics and additional treatment was administered. The patient recovered well and was discharged.

\section{Discussion}

The diagnostic criteria for esophageal-gastric double primary cancer consist of the two esophageal and gastric cancer pathological diagnoses being malignant, the presence of normal mucosa between the two tumors, the exlusion of metastatic cancer, and different tumors must have a unique pathological form (6). Double primary cancers can be divided into simultaneous and metachronous cancers, according to the time of development. A tumor identified within one year of the identification of a novel tumor is termed simultaneous dual primary cancer, if more than one year separates the identification of the lesions, the tumors are termed metachronous double primary cancers.

Simultaneous dual primary cancer is relatively rare, and its treatment is challenging, but if surgeons strictly adhere to the operative indications, radical resection is possible (7). Esophageal-gastric double primary cancer after 5-year survival rate of esophageal cancer is similar to that of simple esophageal cancer surgery or even higher $(8,9)$. Therefore, surgery remains the preferred method of treatment.

In the surgery for the resection of esophageal-gastric double primary cancer, rebuilding the integrity of the digestive tract is the key to a successful procedure. It has been reported that esophageal reconstruction using the colon or jejunum is performed in the majority of patients with esophageal cancer that undergo gastric surgery, while the replacement of the esophagus with the stomach is rare (7). Kwon et al (10) reported a cases of a patient with esophageal-gastric primary cancer and invasive gastric submucosal lesions that were identified during endoscopic resection. Resection of the esophagus was performed, using the stomach to replace the esophagus. At a post-operative follow-up 18 months subsequent to the procedure, the patient was reported to demonstrate a good basic condition.

The surgical procedure of esophageal reconstruction using the upper gastrointestinal tract is generally performed using the stomach or intestines, subsequent to esophagectomy for the excision of esophageal lesions. Due to its simple preparation, reliable blood supply, as well as the decreased anastomosis required, the stomach is the primary choice for upper gastrointestinal reconstruction $(10,11)$. If the exploratory surgery in patients with gastric tumors reveals that the tumor is small, but close to the pyloric ring, surgery to excise the tumor alone may leave residual tumor cells and pyloric stenosis. However, a relatively large region of resection may retain the integrity of the right gastroepiploic artery, which is used to replace the esophagus using the gastrointestinal tract, as it provides the required conditions, thus abandoning the increased number of complications and the higher mortality rate associated with esophageal replacement using the colon and colonic blood supply, such as the unreliable esophageal replacement using the jejunum (12).

During the process of upper gastrointestinal reconstruction, the primary consideration is the blood supply to the esophageal substitute, as the substitute requires good blood supply for post-operative successful rehabilitation and reduction in complications. The key to using a completely dissociated gastric remnant to successfully replace the esophagus in the patient is the complete preservation of the right gastroepiploic artery (Fig. 2), so the size of the gastric antrum cancer and the degree of infiltration should be strictly assessed, which can be assisted by rapid intraoperative frozen sections.

There are two possible surgical methods to achieve remnant stomach and jejunum anastomosis: Billroth or Roux-en-Y 
surgeries. The main advantages of Billroth anastomosis are that it is a simple procedure, with less anastomosis required and a low probability of anastomotic fistula. However, there is often pancreatic and bile reflux. Due to the restriction on the height of the interposition replacement, this procedure is usually used in thoracic anastomosis and anastomosis of the upper end of the aortic arch. The main advantage of the Roux-en-Y type gastric bypass is that the height of the replacement set is higher compared with the Billroth, which significantly reduces pancreatic bile reflux. The Roux-en-Y type procedure also demonstrates more complexity and more anastomotic placement compared with the Billroth procedure, which is most commonly used in cervical anastomosis. The patient possessed middle and lower esophageal cancer, and demonstrated no significant distant invasion. Therefore, the line for esophagogastric anastomosis above the aortic arches was sufficient to remove the tumor completely, so Billroth gastrojejunostomy was performed, with gastroesophageal arch anastomosis above the aortic arches.

Two weeks subsequent to the surgery, upper gastrointestinal imaging of the patient revealed esophagogastric anastomotic leakage, as evidenced by the presence of barium used for imaging in the chest (Fig. 3A). Two possible causes were considered, poor blood flow to the lower stomach or the onset of post-operative pulmonary infection, leading to bad atelectasis. Therefore, an esophageal stent was inserted immediately (Fig. 3B), and effective antibiotics and additional treatment was administered. The patient recovered well and was discharged. As a result, it is recommended that for esophagogastrostomy in esophageal-gastric anastomosis, the posterior or anterior fundus of the stomach, near the greater curvature, is used. Patients should be encouraged to cough effectively post-operatively to promote pulmonary re-expansion, with the administration of effective antibiotics to avoid lung infections and atelectasis.

Patients may undergo gastric antrum tumor resection by endoscopy followed by thoracoscopic resection of the esophageal cancer and cervical esophagogastric anastomosis. The scope of endoscopic resection of the gastric antrum tumor, which is adjacent to pylorus rings, should be noted, as the scope may be too small to complete radical resection of the tumor or it may be too large, which may lead to scarring that results in pyloric stenosis. Therefore, pre-operative endoscopic ultrasound line must be performed to determine the extent of tumor invasion and to accurately choose the surgical procedure.

\section{References}

1. Okamoto N, Ozawa S, Kitagawa Y, Shimizu Y and Kitajima M: Metachronous gastric carcinoma from a gastric tube after radical surgery for esophageal carcinoma. Ann Thorac Surg 77: $1189-1192,2004$

2. Franssen CF, Stegeman CA, Kallenberg CG, Gans RO, De Jong PE, Hoorntje SJ and Tervaert JW: Antiproteinase 3- and antimyeloperoxidase-associated vasculitis. Kidney Int 57: 2195-2206, 2000.

3. Koide N, Adachi W, Koike S, et al: Synchronous gastric tumors associated with esophageal cancer: A retrospective study of twenty-four patients. Am J Gastroenterol 93: 758-762, 1998.

4. Lin ZW, Ge D and Wang Q: Surgical procedures of synchronous double primary esophageal carcinoma associated with gastric carcinoma. Zhongguo Linchuang YiXue Zazhi 18: 483-484, 2011 (In Chinese).

5. Sobin LH, Gospodarowicz M and Wittekind C (eds). UICC International Union Against Cancer. TNM Classification of Malignant Tumours. 7th edition. Wiley-Blackwell, Oxford, 2009.

6. Suzuki S, Nishimaki T, Suzuki T, Kanda T, Nakagawa S and Hatakeyama K: Outcomes of simultaneous resection of synchronous esophageal and extraesophageal carcinomas. J Am Coll Surg 195: 23-29, 2002.

7. Xie SP, Fan GH, Kang GJ, Geng Q, Huang J and Cheng BC: Esophageal reconstruction with remnant stomach: a case report and review of literature. World J Gastroenterol 19: 3169-3172, 2013.

8. Natsugoe S, Matsumoto M, Okumura H, Ishigami S, Uenosono Y, Owaki T, Takao S and Aikou T: Multiple primary carcinomas with esophageal squamous cell cancer: Clinicopathologic outcome. World J Surg 29: 46-49, 2005.

9. Kumagai Y, Kawano T, Nakajima Y, Nagai K, Inoue H, Nara S and Iwai T: Multiple primary cancers associated with esophageal carcinoma. Surg Today 31: 872-876, 2001.

10. Kwon JS, Kim JB, Cho KB, Kim ES, Park KS and Park CK: Gastric tube reconstruction of esophagus for esophageal and stomach carcinomas. Asian Cardiovasc Thorac Ann 20: 600-603, 2012.

11. Davis PA, Law S and Wong J: Colonic interposition after esophagectomy for cancer. Arch Surg 138: 303-308, 2003.

12. Marks JL and Hofstetter WL: Esophageal reconstruction with alternative conduits. Surg Clin North Am 92: 1287-1297, 2012. 